The relation between the estimated dietary intake of PCDD/Fs and levels in blood in a Flemish population (50-65 years)

Peer-reviewed author version

Bilau, Maaike; De Henauw, Stefaan; Schroijen, Carmen; BRUCKERS, Liesbeth; Den Hond, Elly; Koppen, Gudrun; Matthys, Christophe; De Mieroop, Els Van; Keune, Hans; Baeyens, Willy; Nelen, Vera; Van Larebeke, Nik; Willems, Jan L. \& Schoeters, Greet (2009) The relation between the estimated dietary intake of PCDD/Fs and levels in blood in a Flemish population (50-65 years). In: ENVIRONMENT INTERNATIONAL, 35(1). p. 9-13.

DOI: 10.1016/j.envint.2008.05.007

Handle: http://hdl.handle.net/1942/9217 
Provided for non-commercial research and education use. Not for reproduction, distribution or commercial use.

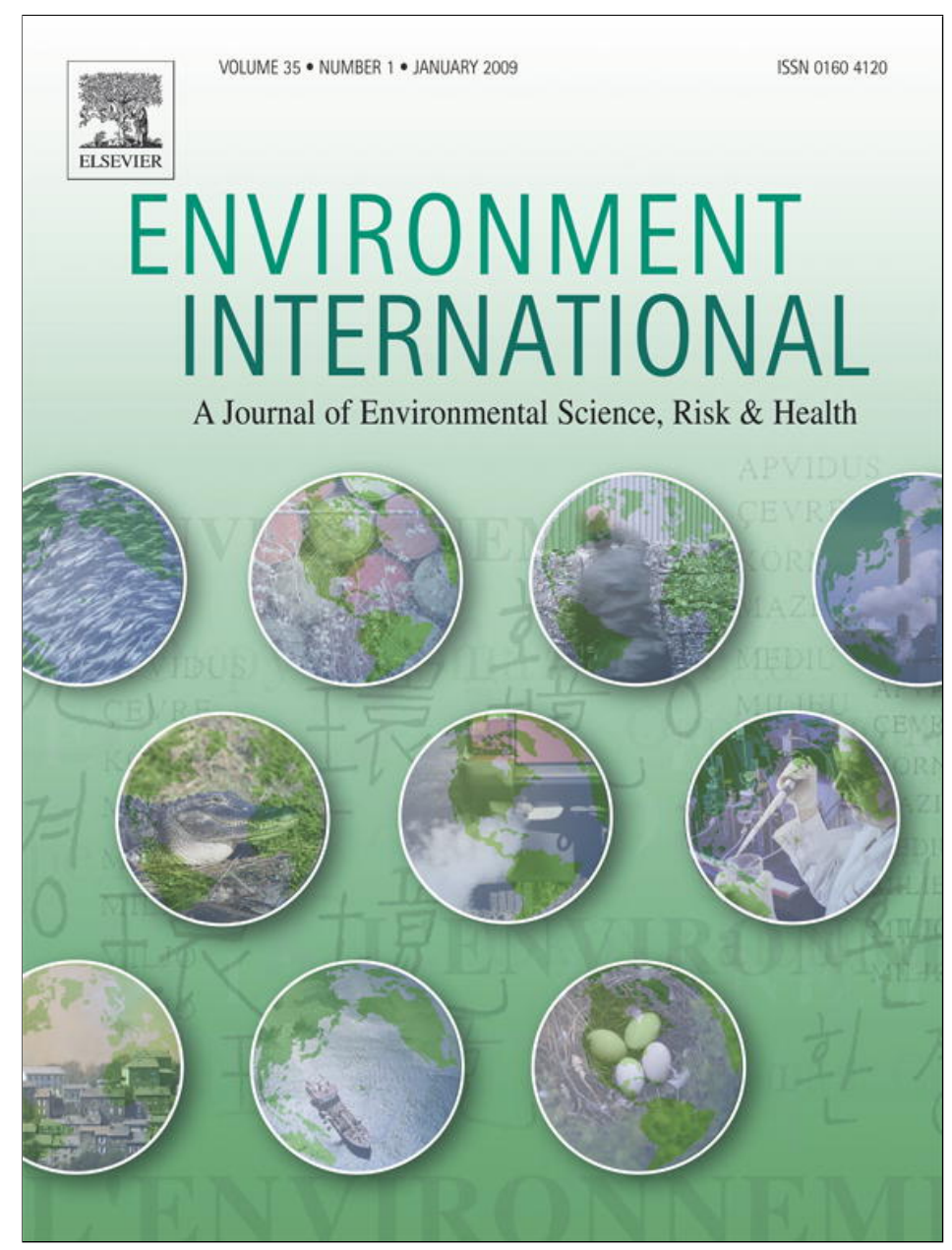

This article appeared in a journal published by Elsevier. The attached copy is furnished to the author for internal non-commercial research and education use, including for instruction at the authors institution and sharing with colleagues.

Other uses, including reproduction and distribution, or selling or licensing copies, or posting to personal, institutional or third party websites are prohibited.

In most cases authors are permitted to post their version of the article (e.g. in Word or Tex form) to their personal website or institutional repository. Authors requiring further information regarding Elsevier's archiving and manuscript policies are encouraged to visit:

http://www.elsevier.com/copyright 


\title{
The relation between the estimated dietary intake of PCDD/Fs and levels in blood in a Flemish population (50-65 years)
}

\author{
Maaike Bilau $^{\mathrm{a}, *}$, Stefaan De Henauw ${ }^{\mathrm{a}, \mathrm{b}}$, Carmen Schroijen ${ }^{\mathrm{c}}$, Liesbeth Bruckers ${ }^{\mathrm{d}}$, Elly Den Hond ${ }^{\mathrm{e}}$, \\ Nik Van Larebeke ${ }^{i}$, Jan L. Willems ${ }^{a}$, Greet Schoeters ${ }^{e, j}$ \\ ${ }^{a}$ Department of Public Health, Ghent University, UZ 2 Blok A, De Pintelaan 185, B-9000 Ghent, Belgium \\ ${ }^{\mathrm{b}}$ Department of Health Sciences, Vesalius, Hogeschool Gent, Keramiekstraat 80, B-9000 Ghent, Belgium \\ c Department of Analytical and Environmental Chemistry, Vrije Universiteit Brussel (VUB), Pleinlaan 2, B-1050 Brussels, Belgium \\ d Center for Statistics, University Hasselt, Agoralaan 1, Building D, B-3590 Diepenbeek, Belgium \\ e Flemish Institute for Technological Research (VITO), Environmental Toxicology, Boeretang 200, B-2400 Mol, Belgium \\ ${ }^{\mathrm{f}}$ Institute of Food, Nutrition and Human Health, Massey University, Private Bag 102 904, North Shore Mail Centre, Auckland, New Zealand \\ g Provincial Institute of Hygiene (PIH) Kronenburgstraat 40, B-2000 Antwerp, Belgium \\ ${ }^{\mathrm{h}}$ Faculty of Political and Social Sciences, University of Antwerp, Sint Jacobstraat 2, B-2000 Antwerp, Belgium \\ i Study Centre of Carcinogenesis and Primary Prevention of Cancer, Ghent University, UZ 3K3, De Pintelaan 185, B-9000 Ghent, Belgium \\ ${ }^{\mathrm{j}}$ University of Antwerp, Universiteitsplein 1, B-2610, Antwerp, Belgium
} Gudrun Koppen ${ }^{\mathrm{e}}$, Christophe Matthys ${ }^{\mathrm{a}, \mathrm{f}}$, Els Van De Mieroop ${ }^{\mathrm{g}}$, Hans Keune ${ }^{\mathrm{h}}$, Willy Baeyens ${ }^{\mathrm{c}}$, Vera Nelen ${ }^{\mathrm{g}}$,

\section{A R T I C L E I N F O}

\section{Article history:}

Received 19 February 2008

Accepted 26 May 2008

Available online 3 July 2008

\section{Keywords:}

Biomonitoring

CALUX

FFQ

Dioxin-like contaminants

Flanders

\begin{abstract}
A B S T R A C T
Dioxin-like activity was measured in the serum of 1425 Flemish men and women via the CALUX assay. The adults, aged between 50 and 65 years, participated in a large biomonitoring program, executed by the Flemish Center of Expertise for Environment and Health between 2002 and 2006. Within the context of this biomonitoring program also dietary intake of dioxin-like contaminants was assessed through a food frequency questionnaire.

The relation between the estimated dietary intake and the dioxin-like activity in serum was evaluated using multivariate analyses: a logistic model was performed on the total population, while a linear regression analysis was done on the subsample with quantifiable dioxin activity levels in serum. Region, gender, age, BMI, smoking status, as well as dietary habits were entered in the model, with dioxin level as an outcome estimate.

Both the logistic and linear model confirmed the contribution of dietary intake to the dioxin activity measured in serum. Also BMI and region were found to be associated with dioxin activity levels.
\end{abstract}

(c) 2008 Elsevier Ltd. All rights reserved.

\section{Introduction}

Flanders, the Dutch-speaking, northern half of Belgium, is one of the most densely populated regions in Europe, with a dense traffic network and industrial activities close to habitation. In order to study the influence of environmental factors on certain health outcomes, a large biomonitoring study was executed by the Flemish Center of Expertise for Environment and Health of the Flemish Community, monitoring several biomarkers of exposure and effect. All public information on the project is available online (www.milieu-engezondheid.be).

One of the biomarkers under study was dioxin activity in serum, measured by the chemical-activated luciferase gene expression (CALUX) assay. The CALUX bioassay uses genetically modified mammalian hepatoma cells that contain a transfected Arylhydrocar-

\footnotetext{
* Corresponding author. Tel.: +32 933224 23; fax: +329 3324994.

E-mail addresses: maaike.bilau@ugent.be, maaikebilau@yahoo.com (M. Bilau).
}

bon Receptor (AhR) responsive luciferase reporter gene which responds to dioxin-like compounds with the induction of luciferase gene expression in a time, dose and chemical specific manner. The serum extract was pretreated to contain only the PCDD/F fraction and no longer the PCB fraction (Van Wouwe et al., 2004). Polychlorinated dibenzo-p-dioxins and dibenzofurans (PCDD/Fs) (further referred to as: dioxins) are a group of lipophilic contaminants, generated unintentionally as by-products from human activities, such as various industrial chemical reactions and combustion processes, including waste incineration. They recycle between air, water, soil, sediments and waste (Fries, 1995). Dioxins in air bind to small size particles and are deposited on fields and plants and are resistant to environmental and biological degradation. They are taken up by animals, concentrate in the lipid fraction of biota and are biomagnified in the food chain. Organisms at higher trophic levels in the food chain contain higher concentrations of lipohilic contaminants. As a consequence, human adipose tissue, serum and breast milk show relatively high levels of dioxins (Safe, 2000). Because of their persistence, resistance to 
degradation and fat solubility, levels of dioxins in the lipid component of body tissues and fluids are a good indicator of cumulative exposure (Arfi et al., 2001).

Increased levels of dioxin-like contaminants are associated with immune deficiency, dermal toxicity, reproductive effects and carcinogenicity as shown in animal and epidemiological studies on accidentally or occupationally exposed cohorts (WHO-ECEH-IPCS, 2000). Dioxin-like compounds have also endocrine disrupting properties. There is a lot of public concern on the health effects of the current environmental levels of dioxin-like compounds.

It is known that dietary intake is the most important source of human exposure: at least $90 \%$ of total exposure to dioxin-like contaminants can be attributed to dietary intake of food items of animal origin (Fries, 1995; Liem et al., 2000; Parzefall, 2002). Within the context of this biomonitoring program, a dietary intake assessment of animal fat has been evaluated, which allowed us to estimate the intake of dioxin-like contaminants through food (Bilau et al. 2008). Since the diet is known to be the most important source of dioxin-like substances in the human body and knowing that executing such a large biomonitoring program is an expensive and timeconsuming task, this biomonitoring project is a unique opportunity to compare the estimated dioxin intake with measured levels of these contaminants in serum of adult men and women, in order to study the potential value of dietary assessment in predicting dioxin concentrations in the human body.

\section{Materials and methods}

\subsection{Study population}

Within the framework of the Flemish Center for Environment and Health, biomonitoring program ran from 2002 till 2006 in eight geographical areas with different types of pollution pressure: two urban areas (city of Ghent and city of Antwerp), four areas with different types of industry (harbour, non-ferrous smelter chemical industry, waste incinerator), a fruit growing area and a rural area. Three age groups were studied: adolescents (14-15 years), mothers and their newborn child, and adults (50-65 years). Only umbillical cord blood was analysed in the subpopulation of the mothers, and no dioxin-like activity was measured in the serum of the adolescents. Therefore, only data on the adult population will be presented.

Between September 2004 and June 2005, adults between 50 and 65 years old were sampled in municipalities, selected at random within the different study areas. The selected municipalities provided addresses of individuals who fell in the correct age range. Individuals were selected via stratified sampling in 3 age groups (50-54 55-59 and 60-65). On the basis of a short questionnaire, it could be determined whether people fulfilled the inclusion criteria. Partners who met the inclusion criteria, could also participate in the study. People who did not meet the inclusion criteria, were randomly replaced by individuals from the same age group and the same sex.

The inclusion criteria for participants were the following: (1) they had to live for at least 5 years in the respective area, (2) they had to be between 50 and 65 years old at the time of the study, and ( 3 ) they had to be able to complete questionnaires in Dutch. All participants signed an informed consent
The participants provided a non-fasting blood and urine sample in which several biomarkers have been measured. They also completed an extensive questionnaire on dietary habits, residence history, education, occupation, lifestyle factors (smoking, hobbies, use of pesticides, ...) and risk perception. Body mass index (BMI) was calculated based on height and bodyweight, measured according to a standardized protocol (WHO, 1995).

In total 1582 adult men and women between 50 and 65 years old were included in the biomonitoring study. For our substudy, 157 subjects had to be excluded because no CALUX measurement has been performed. In total, 1425 subjects ( $48.7 \%$ males) were included in the study presented in this paper.

The biomonitoring study was approved by the Ethical Committee of the University of Antwerp, Belgium.

\subsection{Dietary exposure assessment}

A semi-quantitative Food Frequency Questionnaire (FFQ) was used to estimate the daily intake of lipophilic contaminants (in this case $\mathrm{PCDD} / \mathrm{Fs}$ ) via the consumption of (animal) fat containing food items. Food groups such as meat and meat products, fish and seafood, eggs and dairy products were extensively questioned, as well as added fats (e g baking and frying fat). This FFQ is described in detail elsewhere (Bilau et al., 2008)

Contamination data were provided by the Belgian Federal Agency for the Safety of the Food Chain (www.favv.be). Food items were sampled between 2003 and 2006 and PCDD/Fs levels were determined using the chemical-activated luciferase gene expression (CALUX) assay. The methodology used is described elsewhere (Vanderperren et al., 2004). In order to perform the intake assessment, individual food items (1260 samples) were grouped into 21 food groups, based on fat content and fat origin. These groups were also chosen in view of the feasibility of combining consumption with contamination data. For each group, a mean PCDD/F concentration was calculated. Nondetects were assumed to be half of the limit of quantification (Bilau et al., 2008).

A simple distribution technique was used to estimate the dietary intake of PCDD/Fs, combining the observed distribution of individual food consumption data (food item level) with a point estimate for contaminant concentration (food group level) (Lambe, 2002).

\subsection{Determination of $P C D D / F$ in serum levels}

Concentrations of dioxins and furans (PCDD/Fs) were determined by the CALUX in vitro bioassay in a non-fasting serum sample $(5 \mathrm{~mL})$. The methodology used is described in detail elsewhere (Schroijen et al., 2006; Van Wouwe et al., 2004). Summarily, since PCDD/Fs and PCBs are lipophilic, fat content of serum samples was extracted, using acetone, hexane and a celite column. After evaporation of the eluate, the amount of serum fat was gravimetrically determined. In a second step, other Arylhydrocarbon agonists were eliminated by using well specified mixtures of solvents (hexane, toluene acetone and ethylacetate) and an acid silica column combined with an activated carbon column. In addition, PCDD/Fs were separated from PCBs: only the PCDD/F fractions were analysed in all individual samples via CALUX.

The PCDD/F concentrations were expressed per $\mathrm{mL}$ and per g serum fat. The CALUX analysis was performed with the pGudLuc 6.1 cell line supplied by Xenobiotic Detection Systems Inc (USA).

If dioxin concentrations were too low to be quantified, the samples were recoded to half of the limit of quantification (LOQ) in the data analysis (i.e. 0.03 pg CALUX TEQ/g serum).

This method facilitates the assessment of public health risks due to its high throughput rate and lower cost for the CALUX assay in Belgium compared to chemical analysis such as gas chromatography/mass spectrometry (GC/MS).

\subsection{Statistical analysis}

About 23\% of samples were below the LOQ for CALUX measured in serum. They were considered as half the LOQ i.e. 0.03 pg CALUX TEQ/g serum. Since such a large part

Table 1

Characteristics of the study population (group A)

\begin{tabular}{|c|c|c|c|c|}
\hline & Total $(N=1425)$ & Male $(N=694)$ & Female $(N=731)$ & $p^{\mathrm{b}}$ \\
\hline & Median (IQR) ${ }^{\mathrm{a}}$ & Median (IQR) & Median (IQR) & \\
\hline$\overline{\text { Age (years) }}$ & $57.7(54.0-60.9)$ & $58.5(54.6-61.3)$ & $57.0(53.6-60.5)$ & $<0.001$ \\
\hline BMI $\left(\mathrm{kg} / \mathrm{m}^{2}\right)$ & $26.4(24.1-29.2)$ & $26.9(24.7-29.4)$ & $25.7(23.2-29.0)$ & $<0.001$ \\
\hline$-\mathrm{BMI}<25$ & $36 \%$ & $28 \%$ & $43 \%$ & \\
\hline$-25<\mathrm{BMI}<30$ & $44 \%$ & $51 \%$ & $37 \%$ & \\
\hline$-30<\mathrm{BMI}$ & $20 \%$ & $20 \%$ & $20 \%$ & \\
\hline Estimated intake (pg CALUX TEQ/d) & $146(106-199)$ & $162(120-226)$ & $131(96-179)$ & $<0.001$ \\
\hline Estimated intake (pg CALUX TEQ/kg bw/d) & $1.96(1.43-2.70)$ & $2.01(1.46-2.79)$ & $1.93(1.39-2.61)$ & 0.042 \\
\hline PCDD/F level (pg CALUX TEQ/g serum) & $0.12(0.08-0.17)$ & $0.12(0.08-0.17)$ & $0.12(0.08-0.17)$ & 0.592 \\
\hline PCDD/F level (pg CALUX TEQ/g serum fat) & $23.0(12.0-32.7)$ & $22.4(11.8-32.1)$ & $23.4(12.3-33.5)$ & 0.399 \\
\hline Smoking habits & & & & $<0.001$ \\
\hline - Current smokers & $18 \%$ & $21 \%$ & $14 \%$ & \\
\hline - Former smokers & $37 \%$ & $48 \%$ & $25 \%$ & \\
\hline - Never smokers & $45 \%$ & $29 \%$ & $61 \%$ & \\
\hline
\end{tabular}

\footnotetext{
${ }^{a}$ IQR interquartile range.
}

b Differences between sexes (Mann-Whitney $U$ test). 
Table 2

Spearman Rank Correlations ( $p$-values) between estimated dietary intake of PCDD/Fs (pg CALUX TEQ/d) for different food groups and CALUX levels in serum (pg CALUX TEQ/ g serum) for group B

\begin{tabular}{llcl}
\hline PCDD/F intake via & $\begin{array}{l}\text { Total group } \\
(N=1092)\end{array}$ & $\begin{array}{l}\text { Change in dietary } \\
\text { habits }(N=395)\end{array}$ & $\begin{array}{l}\text { No change in dietary } \\
\text { habits }(N=677)\end{array}$ \\
\hline fish and seafood & $0.072(0.018)$ & $0.038(0.457)$ & $0.115(0.003)$ \\
dairy products & $0.035(0.252)$ & $-0.027(0.600)$ & $0.071(0.065)$ \\
meat and meat products & $0.075(0.014)$ & $-0.012(0.814)$ & $0.124(0.001)$ \\
eggs & $0.056(0.062)$ & $0.039(0.444)$ & $0.072(0.060)$ \\
added fats & $0.007(0.809)$ & $-0.057(0.260)$ & $0.061(0.115)$ \\
total diet & $0.086(0.004)$ & $0.026(0.612)$ & $0.145(<0.001)$ \\
\hline
\end{tabular}

of the CALUX levels were below the LOQ different analyses were performed on two groups of the population: 1) group A, the whole study population $(N=1425)$ and 2 ) group $B$, a subsample of the study population $(N=1092)$ namely the subjects with quantifiable levels of PCDD/Fs in their serum. Normality was tested for both groups (Kolmogorov-Smirnov) and descriptive statistics were analysed for the whole study population (median; interquartile range).

\subsubsection{Univariate analysis}

Non-parametric tests were used to study the relation between the estimated dietary intake of PCDD/Fs and measured dioxin activity levels (CALUX) in serum of group A. Mann-Withney $U$ test was used to test for differences between both sexes, while Kruskall-Wallis (KW) test was used to test for differences between normal, overweight and obese individuals.

Spearman rank correlation was also performed on group B comparing correlations between estimated dietary intake and measured CALUX levels for a group of participants that have declared that their dietary habits have, versus have not, changed in the past 5 years.

\subsubsection{Multivariate analysis}

Since PCDD/F serum levels in adult men and women are the result of a continuous accumulation of these substances throughout the entire life, several influencing factors have to be taken into account in a multivariate model.

In a first model, the 75th percentile (P75) of CALUX in serum was used to divide the whole study population $(N=1425)$ in a highly exposed group (above P75) and a lower exposed group (below P75). A logistic regression was used to investigate the association of sex, age, region, dietary intake, BMI and smoking status with PCDD/F serum levels above P75 as the dependent variable, computing the odds ratios (ORs) as measures of association. This analysis was also performed for two subgroups: in order to compare the results of participants who have declared that their dietary habits have, versus have not, changed in the past 5 years.

In a second model, linear regression analysis was done on a subsample of the population $(N=1092)$ with quantifiable dioxin levels in order to investigate the possibility that measured CALUX levels in serum could be predicted by dietary habits, sex, age, region, BMI and smoking status.

Table 3

Odds ratio (OR) and 95\% confidence interval (CI) as a result of multivariate logistic regression analysis with dioxin levels (pg CALUX TEQ/g serum) above P75 as the response variable (group $\mathrm{A} ; \mathrm{N}=1425$ )

\begin{tabular}{lllr}
\hline & OR & 95\% CI & $p$-value \\
\hline Quartiles of dietary intake (pg PCDD/Fs TEQ/d) & & & 0.020 \\
Q1 & 0.619 & $0.431-0.890$ & 0.010 \\
Q2 & 0.606 & $0.424-0.865$ & 0.006 \\
Q3 & 0.778 & $0.554-1.095$ & 0.150 \\
Q4 & 1 & & \\
Region & & & $<0.001$ \\
$\quad$ City of Ghent & 0.396 & $0.256-0.612$ & $<0.001$ \\
$\quad$ City of Antwerp & 1.585 & $1.175-2.138$ & 0.003 \\
Harbour & 0.918 & $0.654-1.289$ & 0.622 \\
$\quad$ Non-ferrous smelter (Olen) & 0.961 & $0.693-1.334$ & 0.814 \\
$\quad$ Chemical industry (Albert Canal area) & 0.744 & $0.523-1.059$ & 0.101 \\
Waste incinerator & 1.352 & $0.996-1.835$ & 0.053 \\
Fruit growing area & 1.372 & $0.985-1.912$ & 0.061 \\
Rural area & 1.307 & $0.959-1.783$ & 0.091 \\
Sex (female as reference) & 0.891 & $0.680-1.169$ & 0.405 \\
Age & 0.960 & $0.969-1.030$ & 0.999 \\
BMI & 1.035 & $1.004-1.066$ & 0.025 \\
Smoking status & & & 0.542 \\
$\quad$ Current smoker & 0.851 & $0.594-1.221$ & 0.381 \\
Former smoker & 0.868 & $0.650-1.160$ & 0.339 \\
$\quad$ Never smoker & 1 & & \\
Constant & 0.081 & & 0.011 \\
\hline
\end{tabular}

Data were analysed using the SPSS $₫$ software package (version 15.0). The level of statistical significance was set at $p \leq 0.05$.

\section{Results}

\subsection{Description of the population}

Main characteristics of the study population $(N=1425)$, measured PCDD/F serum concentrations and estimated PCDD/F intakes, are presented in Table 1. Regarding BMI, 511 participants (36\%) have a normal weight, 626 participants (44\%) are overweight $(25<\mathrm{BMI}<30)$ and $287(20 \%)$ are obese.

\subsection{Univariate analysis}

In group A (i.e. the whole study population) no differences were seen in CALUX levels between men and women (Mann-Whitney $U$ ). However, CALUX levels (pg TEQ/g serum) were significantly different between normal $(B M I<25)$, overweight $(25<\mathrm{BMI}<30)$ and obese participants $(30<\mathrm{BMI})(\mathrm{KW} ; p=0.036)$. The median CALUX levels were respectively $0.113,0.122$ and $0.122 \mathrm{pg}$ CALUX TEQ/g serum. Also the estimated intake (pg TEQ/bw kg/d as well as pg TEQ/d) was different for normal, overweight and obese participants (KW; $p<0.001$ ). Their median estimated intakes were respectively 2.13, 1.96 and $1.70 \mathrm{pg}$ CALUX TEQ/kg bw/d and 136, 151 and $152 \mathrm{pg}$ CALUX TEQ/d.

To compare the estimated dietary intake of PCDD/Fs with CALUX levels in serum, Spearman Rank Correlation was determined $(r=0.086, p=0.004)$ in group $\mathrm{B}$, the group of participants with quantifiable levels of PCDD/Fs in their serum. Around $40 \%$ of that group stated that their dietary habits had been changed in the last 5 years. Spearman Rank Correlations were statistically different for people with or without changed dietary habits (see Table 2).

\subsection{Multivariate analysis}

In a first stage, a logistic model was performed with CALUX levels in serum above the P75 as a response variable and dietary intake (quartile 1,2 and 3 vs the highest quartile), region (average CALUX level as a reference), sex (female as a reference), smoking status (never smoker as a reference), age (continuous variable) and BMI (continuous variable) as predictive variables (group $\mathrm{A}, N=1425$ ). Results for this model are presented in Table 3.

Odds ratios (OR) were statistically significant for dietary intake, region and BMI. The OR for having CALUX levels above the P75 was significantly lower for participants in the first and second quartile of estimated dietary intake. The city of Ghent had a 60\% lower chance for having CALUX levels above the P75 than other regions, whereas the city of Antwerp had a significantly higher chance to have CALUX levels above the P75. There was also a significant increase of $3.5 \%$ per unit increase of BMI to have CALUX levels above the P75. No statistical significant relation was found with age, sex or smoking status.

Similar results were found when analysis was performed on the smaller subgroup of participants who stated that their dietary habits have not been changed in the past 5 years (OR (95\% CI) $0.506(0.317-0.806)$ for first quartile of dietary intake). However, ORs were not statistically significant for dietary intake for participants stating that they have changed their dietary habits.

\section{Table 4}

Multivariate linear regression: associations between dioxin CALUX levels in serum and selected explanatory variables among adults with quantifiable CALUX activity (group B)

\begin{tabular}{|c|c|c|c|}
\hline & $\begin{array}{l}\text { Unstandardized } \\
\text { beta }\end{array}$ & $\begin{array}{l}\text { Standardized } \\
\text { beta }\end{array}$ & $p$-value \\
\hline \multicolumn{4}{|l|}{ Dietary intake (pg PCDD/Fs TEQ/d) } \\
\hline Intake via fish/seafood & 0.00011 & 0.064 & 0.039 \\
\hline Intake via dairy & 0.00004 & 0.013 & 0.678 \\
\hline Intake via meat/meat products & 0.00044 & 0.068 & 0.039 \\
\hline Intake via egg & 0.00030 & 0.019 & 0.532 \\
\hline Intake via added fats ${ }^{\mathrm{a}}$ & -0.00007 & -0.027 & 0.431 \\
\hline \multicolumn{4}{|l|}{ Region $^{\mathrm{b}}$} \\
\hline City of Antwerp & 0.03013 & 0.134 & 0.002 \\
\hline Harbour & 0.03501 & 0.144 & 0.001 \\
\hline Non-ferrous smelter (Olen) & 0.02433 & 0.105 & 0.015 \\
\hline Chemical industry (Albert Canal area) & 0.01114 & 0.049 & 0.261 \\
\hline Waste incinerators & 0.03339 & 0.147 & 0.001 \\
\hline Fruit growing area & 0.02739 & 0.112 & 0.008 \\
\hline Rural area & 0.04466 & 0.194 & $<0.001$ \\
\hline $\operatorname{Sex}^{\mathrm{b}}$ & 0.00822 & 0.053 & 0.116 \\
\hline Age & 0.00002 & 0.001 & 0.977 \\
\hline BMI & 0.00139 & 0.073 & 0.018 \\
\hline Smoking status ${ }^{\mathrm{b}}$ & 0.00124 & 0.008 & 0.804 \\
\hline Constant & & & 0.100 \\
\hline
\end{tabular}

${ }^{\mathrm{a}}$ Added fats $=$ spreads, baking and frying fats.

b City of Ghent (region), female (sex) and never smoking (smoking status) are references in the model. 
In a second phase, linear regression was performed on the subjects with quantifiable levels of dioxin activity in their serum only (group B; $N=1092$ ) with CALUX levels (pg TEQ/g serum) as the outcome variable. The $R$ square of this model was 0.047 . Coefficients and significance level of the introduced parameters are presented in Table 4.

\section{Discussion}

It is widely accepted that the diet is responsible for more than $90 \%$ of the exposure to dioxin-like contaminants in the general population (Fries 1995; Liem et al., 2000; Päpke, 1998; Parzefall 2002). Therefore, a dietary intake assessment is often performed as an exposure and risk estimate for the general population regarding this type of contaminants (Bilau et al., 2008; Bocio and Domingo, 2005; Charnley and Doull, 2005; Darnerud et al., 2006; Fattore et al., 2006; Kiviranta et al., 2004; Sasamoto et al., 2006; Taioli et al., 2005; Vrijens et al., 2002).

Measurement of the concentration of dioxin-like contaminants in adipose tissue, human breast milk or serum constitutes another exposure and surrogate risk estimate. The association however between both exposure estimates, e.g. the serum concentrations, and the estimated dietary intake was mainly studied in specific subgroups of the population, such as recreational fishermen (Chen et al., 2003; Lee et al., 2006b; Turyk et al., 2005). Similar analyses in the general population are less often reported (Arfi et al., 2001; Hauser et al., 2005; Lee et al., 2007). Although not the primary target of the biomonitoring study, we found it of interest to study this association in our study population. The original aim of assessing the dietary exposure to dioxin-like contaminants, was to use the results of this intake estimation as an adjusting factor in the relations under study in the biomonitoring program.

\section{1. $P C D D / F$ levels measured in serum}

The reported median level of dioxin CALUX activity in serum was $23 \mathrm{pg}$ CALUX TEQ/g serum lipid. In a previous Flanders Environmental and Health Study, PCDD/Fs were determined in serum fat of 200 women between 50 and 65 years using GC-HRMS (Koppen et al., 2002). The results reported were higher ( $48 \mathrm{pg} \mathrm{TEQ} / \mathrm{g}$ serum fat) but a direct comparison remains difficult as the analytical methodology was different - a chemical analysis versus the CALUX test. According to Van Wouwe et al. (2004) and based on 341 plasma samples, a significant correlation was established between the bioassay and chemical method $(R=0.64)$. However, a proportional systematic error (CALUX was higher than GC-HRMS) was observed when the results obtained with the CALUX bioassay were regressed with the results from the GC-HRMS analyses. The limit of quantification (LOQ) used to calculate TEQ values from the GC-HRMS determinations, the use of the relative potency values instead of the toxic equivalent factor and the potential of CALUX bioassay to measure all compounds with affinity for the AhR may partly explain this proportional systematic error. Nevertheless, the present results suggest that the CALUX bioassay could be a promising valid screening method for human blood plasma analyses.

Overall, the levels reported in this paper, were equal to or lower than results found in other countries (Arisawa et al., 2005; Chen et al., 2007; Lee et al., 2006a). However, in countries where a younger population were studied, levels were somewhat lower (Cerna et al., 2007), which is easily explained by differences in age.

\subsection{Factors influencing $P C D D / F$ levels}

The present analysis confirmed that dietary intake contributes to the dioxin CALUX activity of serum. Logistic as well as linear regression models showed a statistically significant relation with dietary exposure. In the linear regression model, it was shown that the intake of meat and meat products on the one hand and fish and seafood on the other hand, have the largest influence within the diet.
Both food groups were expected to have an important contribution because of the large amounts consumed (meat/meat products) and the relatively high contaminant levels (fish/seafood) (Bilau et al., 2008; Vrijens et al., 2002).

The region in which people have been living for at least 5 years also is an explanatory factor. Participants living in the city of Ghent seem to have the lowest CALUX levels in serum, while the participants living in the city of Antwerp seem to have higher CALUX levels. An explanation for this difference is, however not obvious. Also the harbour and rural area have significantly higher CALUX levels. The region around waste incinerators as well as the fruit growing area showed a higher CALUX level, although not statistically significant. There might be some differences in the prevalence and/or amount of consumption of locally grown products (home grown vegetables, but also home produced eggs). The influence of the consumption of locally produced foods on serum levels of lipophilic contaminants, was also reported in other studies (Donato et al., 2006). However, the questionnaire did not provide enough detailed information to study this relationship adequately. It would be interesting to include in more detail the consumption of locally grown products in future questionnaires.

For individuals with a higher BMI, higher levels of dioxin activity in blood (pg CALUX TEQ/g serum) were found. However, this could not be explained by differences in dietary intake per kg bodyweight. The relation between BMI, dietary intake and CALUX levels in serum is a complex relation: people with a higher BMI are likely to have a higher dietary fat intake and thus a higher intake of fat-soluble contaminants. However, because of the higher amount of body fat expected, contaminants can be distributed over a larger volume of adipose tissue.

For bioaccumulative contaminants, such as $\mathrm{PCDD} / \mathrm{Fs}$, age is an important predictor of body burden. However, the age range in our study population was rather narrow (50-65 years). Moreover, body burden of all participants was assumed to have already reached the steady state level. Therefore, a relation between age and the serum concentration was not expected in this study population.

It was reported that smoking influences the metabolism of lipophilic contaminants. In our study, the chance to have a CALUX level above the P75 for current smokers was not significantly different, but chances for current and former smokers to have CALUX levels above the P75 tend to be smaller.

\subsection{Methodological considerations}

\subsubsection{Calux measurement in serum}

Although measuring PCDD/F levels in adipose tissue is known to be the best parameter for body burden, it was impossible to use this invasive measurement in a large biomonitoring study (Allam and Lucena, 2001). Analysing dioxin levels in serum by measuring CALUX activity is a reasonable proxy for estimating the PCDD/F body burden, certainly if no other media (such as adipose tissue) are present (Whitcomb et al., 2005).

The CALUX bioassay measures an activity level rather than an exact concentration of congeners, although with the clean-up procedure used in this study, a strong correlation between CALUX and gas chromatography-high resolution mass spectrometry (GC-HRMS) results was observed (Van Wouwe et al., 2004). Moreover, there might be some influence of short term dietary intake, since participants did not have to be fasting at the moment the blood sample was taken. Consequently it was possible that the levels in serum were not always in equilibrium with the levels in adipose tissue at the moment of sampling.

\subsubsection{Dietary intake assessment}

On the other hand, the intake assessment is also prone to measurement error. It is, above all, an almost impossible task, to assess the life-long dietary habits of individuals at an age of 50 to 
65 years old. As a proxy for life-long dietary intake, participants were asked to report dietary habits of the year before the study. Although one year is only a relatively short period when compared to the age reached at the moment of blood sampling, this is a long period when one considers the fact that the FFQ depends completely on the memory of participants.

Also, certain changes in dietary habits could have occurred throughout their life, resulting in incorrect estimation of life-long dietary intake. This was confirmed: almost $30 \%$ of the study population stated that their dietary habits have changed within the last 5 years. Of those 395 participants, 60\% stated that their consumption of fish and seafood has increased over the last 5 years, while $81 \%$ stated that their consumption of meat and meat products has decreased over the last 5 years. The majority of the participants with changed dietary habits (54\%) stated that their egg consumption decreased as well. However, in order to study this in detail, more information would be needed. Therefore, statistical analyses were done on the group who stated not to have changed their diet: the associations found were stronger, despite the smaller population under study. This uncertainty on dietary exposure may, in part, explain the relatively low $\mathrm{R}^{2}$ values in the regression models.

\subsubsection{Contaminant data}

Also the contaminant levels in food items were affected by uncertainties, due to the sampling strategy, the methodology used and the evolution in time. For all participants the same contaminant levels were used, although it was possible that some participants consumed on average food items with higher contaminant levels than did others (e.g. participants consuming more or more often locally grown eggs).

\section{Conclusion}

Total dietary exposure (predominantly exposure via meat, meat products, fish and seafood), BMI and region were found to be associated with concentrations of PCDD/F, measured by CALUX in non-fasting serum samples of Flemish adults between 50 and 65 years old. However, estimated food intake in a general population with a rather homogenous dietary pattern seemed a less important factor in explaining the variation in dioxin activity in serum by CALUX compared to BMI and region, although the diet is the main contributor of PCDD/F exposure.

\section{Acknowledgements}

The authors would like to thank the dieticians Mrs Mia Bellemans and Mrs Mieke De Maeyer for their important contribution to the fieldwork and the data input. We also would like to thank the Federal Agency for the Safety of the Food Chain (FAVV) for access to the analytical data. The intake assessment performed is solely the responsibility of the authors and do not engage the FAVV.

The study was commissioned, financed and steered by the Ministry of the Flemish Community (Department of Science, Department of Public Health and Department of Environment), without any responsibility for the scientific content.

\section{References}

Allam MF, Lucena RA. Breast cancer and PCBs: true or false association? Eur J Cancer Prev 2001;10:539-40.

Arfi C, Seta N, Fraisse D, Revel A, Escande JP, Momas I. Dioxins in adipose tissue of nonoccupationally exposed persons in France: correlation with individual food exposure. Chemosphere 2001;44:1347-52.

Arisawa $\mathrm{K}$, Takeda H, Mikasa $\mathrm{H}$. Background exposure to PCDDs/PCDFs/PCBs and its potential health effects: a review of epidemiologic studies. J Med Invest 2005;52:10-21.

Bilau M, Matthys C, Baeyens W, Bruckers L, De Backer G, Den Hond E, et al. Dietary exposure to dioxin-like compounds in three age groups: results from the Flemish environment and health study. Chemosphere 2008;70:584-92.
Bocio A, Domingo JL. Daily intake of polychlorinated dibenzo-p-dioxins/polychlorinated dibenzofurans (PCDD/PCDFs) in foodstuffs consumed in Tarragona, Spain: a review of recent studies (2001-2003) on human PCDD/PCDF exposure through the diet. Environ Res 2005;97:1-9.

Cerna M, Kratenova J, Zejglicova K, Brabec M, Maly M, Smid J, et al. Levels of PCDDs, PCDFs, and PCBs in the blood of the non-occupationally exposed residents living in the vicinity of a chemical plant in the Czech Republic. Chemosphere 2007;67: S238-46.

Charnley G, Doull J. Human exposure to dioxins from food, 1999-2002. Food Chem Toxicol 2005:43:671-9.

Chen HL, Lee CC, Liao PC, Guo YL, Chen CH, Su HJ. Associations between dietary intake and serum polychlorinated dibenzo- $p$-dioxin and dibenzofuran (PCDD/F) levels in Taiwanese. Environ Res 2003;91:172-8.

Chen HL, Su HJ, Lee CC. Association between tofu intake and serum polychlorinated dibenzo- $p$-dioxins and dibenzofurans $(\mathrm{PCDD} / \mathrm{Fs})$ in the elderly Taiwanese. Environ Int 2007;33:265-71.

Darnerud PO, Atuma S, Aune M, Bjerselius R, Glynn A, Grawe KP, et al. Dietary intake estimations of organohalogen contaminants (dioxins, PCB, PBDE and chlorinated pesticides, e.g. DDT) based on Swedish market basket data. Food Chem Toxicol 2006:44:1597-606.

Donato F, Magoni M, Bergonzi R, Scarcella C, Indelicato A, Carasi S, et al. Exposure to polychlorinated biphenyls in residents near a chemical factory in Italy: the food chain as main source of contamination. Chemosphere 2006;64:1562-72.

Fattore E, Fanelli R, Turrini A, di Domenico A. Current dietary exposure to polychlorodibenzo-p-dioxins, polychlorodibenzofurans, and dioxin-like polychlorobiphenyls in Italy. Mol Nutr Food Res 2006;50:915-21.

Fries GF. A review of the significance of animal food products as potential pathways of human exposures to dioxins. J Anim Sci 1995;73:1639-50.

Hauser R, Williams P, Altshul L, Korrick S, Peeples L, Patterson Jr DG, et al. Predictors of serum dioxin levels among adolescent boys in Chapaevsk, Russia: a cross-sectional pilot study. Environ Health 2005;4:8.

Kiviranta H, Ovaskainen ML, Vartiainen T. Market basket study on dietary intake of PCDD/Fs, PCBs, and PBDEs in Finland. Environ Int 2004;30:923-32.

Koppen G, Covaci A, Van Cleuvenbergen R, Schepens P, Winneke G, Nelen V, et al. Persistent organochlorine pollutants in human serum of 50-65 years old women in the Flanders Environmental and Health Study (FLEHS). Part 1: concentrations and regional differences. Chemosphere 2002;48:811-25.

Lambe J. The use of food consumption data in assessments of exposure to food chemicals including the application of probabilistic modelling. Proc Nutr Soc 2002;61:11-8.

Lee CC, Guo YL, Kuei CH, Chang HY, Hsu JF, Wang ST, et al. Human PCDD/PCDF levels near a pentachlorophenol contamination site in Tainan, Taiwan. Chemosphere 2006a;65:436-48

Lee CC, Lin WT, Liao PC, Su HJ, Chen HL. High average daily intake of PCDD/Fs and serum levels in residents living near a deserted factory producing pentachlorophenol (PCP) in Taiwan: influence of contaminated fish consumption. Environ Pollut 2006b;141:381-6.

Lee SA, Dai O Zheng W, Gao YT, Blair A, Tessari JD, et al. Association of serum concentration of organochlorine pesticides with dietary intake and other lifestyle factors among urban Chinese women. Environ Int 2007;33:157-63.

Liem AK, Fürst P, Rappe C. Exposure of populations to dioxins and related compounds. Food Addit Contam 2000;17:241-59.

Päpke O. PCDD/PCDF: human background data for Germany, a 10-year experience. Environ Health Perspect 1998;106(Suppl 2):723-31.

Parzefall W. Risk assessment of dioxin contamination in human food. Food Chem Toxicol 2002;40:1185-9.

Safe SH. Endocrine disruptors and human health - is there a problem? An update. Environ Health Perspect 2000;108:487-93.

Sasamoto T, Ushio F, Kikutani N, Saitoh Y, Yamaki Y, Hashimoto T, et al. Estimation of 1999-2004 dietary daily intake of PCDDs, PCDFs and dioxin-like PCBs by a total diet study in metropolitan Tokyo, Japan. Chemosphere 2006;64:634-41.

Schroijen C, Van Wouwe N, Sanctorum H, Goeyens L, Baeyens W. Calux analysis in small amounts of human serum. Organohalogen Compounds 2006;68:2511-4.

Taioli E, Marabelli R, Scortichini G, Migliorati G, Pedotti P, Cigliano A, et al. Human exposure to dioxins through diet in Italy. Chemosphere 2005;61:1672-6.

Turyk M, Anderson HA, Hanrahan LP, Falk C, Steenport DN, Needham LL, et al. Relationship of serum levels of individual PCB, dioxin, and furan congeners and DDE with Great Lakes sport-caught fish consumption. Environ Res 2005.

Van Wouwe N, Windal I, Vanderperren H, Eppe G, Xhrouet C, Massart AC, et al. Validation of the CALUX bioassay for PCDD/F analyses in human blood plasma and comparison with GC-HRMS. Talanta 2004;63:1157-67.

Vanderperren H, Van Wouwe N, Behets S, Windal I, Van Overmeire I, Fontaine A. TEQvalue determinations of animal feed; emphasis on the CALUX bioassay validation. Talanta 2004;63:1277-80.

Vrijens B, De Henauw S, Dewettinck K, Talloen W, Goeyens L, De Backer G, et al. Probabilistic intake assessment and body burden estimation of dioxin-like substances in background conditions and during a short food contamination episode. Food Addit Contam 2002;19:687-700.

Whitcomb BW, Schisterman EF, Buck GM, Weiner JM, Greizerstein H, Kostyniak PJ. Relative concentrations of organochlorines in adipose tissue and serum among reproductive age women. Environ Toxicol Pharmacol 2005;19:203-13.

WHO. Physical status: the use and interpretation of anthropomethry. 854. Geneva, Switserland: World Health Organization; 1995.

WHO-ECEH-IPCS. Consultation on assessment of the health risks of dioxins; reevaluation of the tolerable daily intake (TDI): executive summary. Food Addit Contam 2000;17:223-40. 